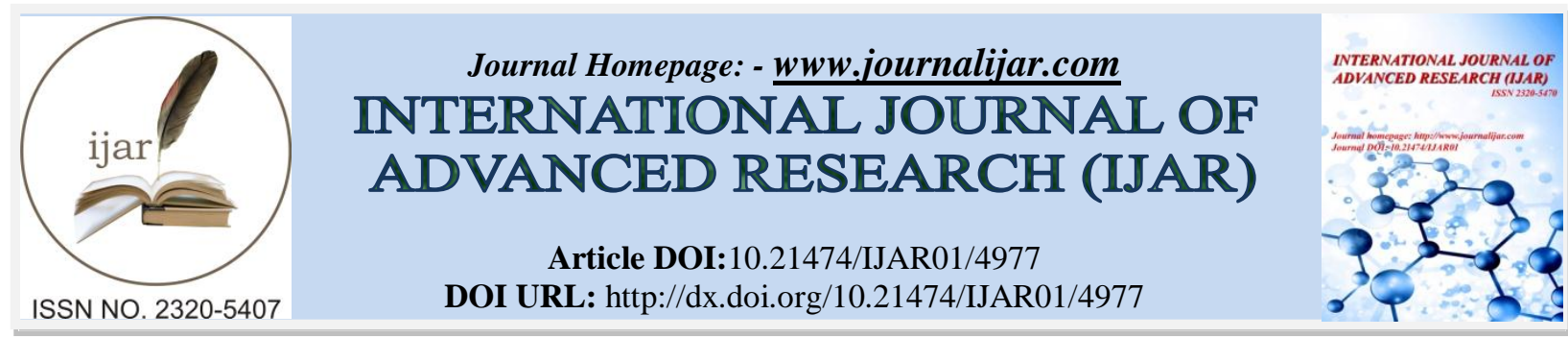

RESEARCH ARTICLE

\title{
ATAXIA TELANGIECTASIA: A CASE REPORT.
}

Khaire $\mathbf{P} \mathbf{B}^{1}$, Dahyalkar A A ${ }^{2}$, Patil $M A^{3}$, Kachhap $\mathbf{R}^{4}$ and Kasliwal $C A^{2}$.

1. Associate Prof., Department of Pediatrics, Govt. Medical College, Aurangabad.

2. Assistant Prof., Department of Pediatrics, Govt. Medical College, Aurangabad.

3. JR2, Department of Pediatrics, Govt. Medical College, Aurangabad.

4. JR3- Department of Pediatrics, Govt. Medical College, Aurangabad.

\section{Manuscript Info}

\section{Manuscript History}

Received: 27 May 2017

Final Accepted: 29 June 2017

Published: July 2017

Key words:-

Ataxia-telangectasia;neurodegenerative disease.

\section{Abstract}

Ataxia-telangiectasia (A-T) is a rare hereditary neurodegenerative disorder. Ataxia and telangiectasias are the hallmarks of the disease. Due to its wide clinical heterogeneity, it often leads physicians to an incorrect or missed diagnosis, and insight into this rare disease is important. There is no gold standard diagnostic test and diagnosis relies on clinical evaluation, exclusion of similar conditions, and supportive laboratory tests. More than $99 \%$ of individuals with classic A-T have mutations in ATM, the only gene known to be associated with ataxia-telangiectasia.We report 10 year old boy who was presented with respiratory tract infections, unsteady gait, frequent falls and telangectasias of the eyes. He also has had frequent episodes of respiratory tract infections.

Copy Right, IJAR, 2017. All rights reserved.

\section{Introduction:-}

Ataxia telangiectasia (AT) is a rare multisystem, neurodegenerative genetic disorder. (1)Due to its wide clinical heterogeneity, it often leads physicians to an incorrect or missed diagnosis, and insight into this rare disease is important. The syndromewas not mentioned until 1941 when Louis-Bar reported a case of a 9-year-old child. (2) Boder et al recognized the familial incidenceproposing an autosomal recessive mode of inheritance for the disease.(1)Theataxiatelangiectasia mutated (ATM) kinase initiates a well characterized response to DNA damage, resulting in arrest of cell-cycle, DNA repair, or apoptosis.(2) A-T has led to much speculation about additional pathogenic mechanisms including oxidative stress. How ATM is involved in oxidative stress management remains unclear as do other potential roles in cellular homeostasis in the absence of DNA damage.(2) The incidence of ataxia-telangiectasia is about 1 case in 100,000 births.(3) The frequency of ataxiatelangiectasia mutant alleles heterozygosity was reported to be 1.4- $2 \%$ of the general population.(2-3)Ataxia-telangiectasia occurs equally among males and females.

Case Report:-

A 10 years old boy who is the first issue of non consanguineous parents completely immunized according to EPI schedule was admitted to Government Medical College Hospital Aurangabad, on 26.01.2010 with the complaints of generalized weakness and difficulty in walking from 3 years of life and 


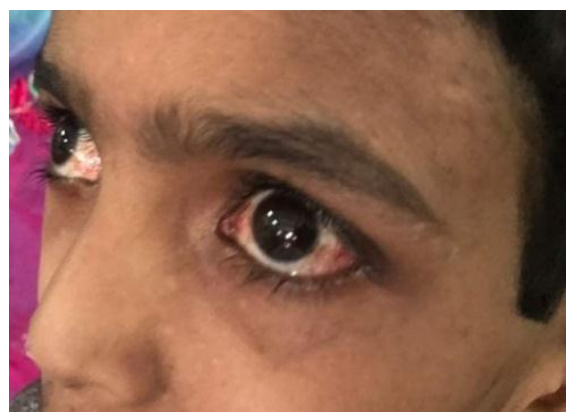

Fig 1

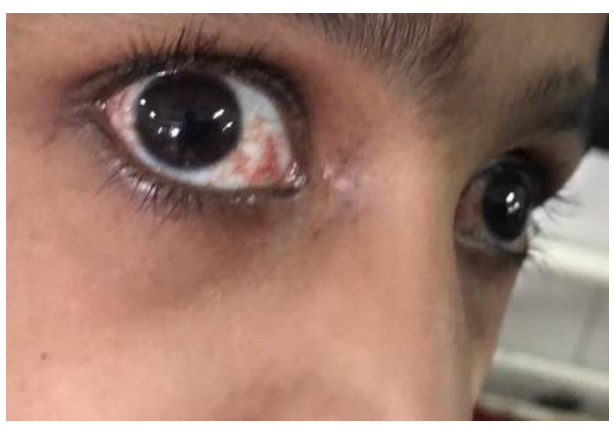

Fig 2

Fig.1 \& Fig.2:-showing bilateral bulbar conjuntival telangectasia“

breathlessnesssince 5 days. The patient initially could try to walk but later on he fell on the ground as hedeveloped imbalance in walking that gradually involved also in standing capacity within one month. He also developed congestions of the conjunctiva that has appeared at age 1year. The congestions werefirst noted in the interpalpebral bulbar conjunctiva away from the limbus. Pasthistory - suggestive of frequent respiratory infections. Birth history was normal.

On general examination, gait was ataxic. The patient was found anemic. Congestion was seen in botheyes that is radiating from the both corners to limbus. The patient was conscious and cooperative. On CNS examination, cranial nerves were normal. Therewere hypotonia in all four limbs which was more marked in lower limbs with diminished jerks and flexor plantar reflexes. Sensory function was intact. Contracture were present .Cerebellarfunction was abnormal showed slurred speech, intention tremor, past pointing and dysdiadochokinesia. On investigation, complete blood count, lipid profile, LFT, chest x-ray, serum electrolytes, ultra sonogram of whole abdomen, serumIg $\mathrm{G}$ and ECG showed normal results.

Serum $\alpha$-fetoprotein was increased that is $1250 \mathrm{ng} / \mathrm{L}$. serum IG A level decreased $0.5 \mathrm{gm} / 1$. There wasno specific treatment was provided for AT.

\section{Discussion:-}

In our index case, there was respiratory infections, progressive cerebellar ataxia, bulbar telangiectasias, raised serum alpha-fetoprotein and decreased IG Alevel .These clinch us to reach the diagnosis of Ataxia telangiectasia. In general, Ataxiatelangiectasia (AT) is a multisystem disorder characterized by progressive neurologic impairment, cerebellar ataxia, progressive immunodeficiency, ocular and cutaneoustelangiectasia, increased risk of lymphoreticular malignancy and hypersensitivity to ionizing radiation. $(1,3,4)$ Telangiectasia can also appear on sun exposed areas of skin such as face and ears, occasionally arise in liver and lungs.(5) Both humoral\& cellular immunity are impaired in patients with AT. (5)The most common immunologic abnormality is the absence ordecreased level of serum \& salivary level ofIgA. (5)This deficiency has been found in 50 to 80 percent of these patients. Patients with AT have an increased susceptibility to sinopulmonary infection, x-ray hypersensitivity, and predisposition tomalignancy.Chromosomal abnormalities in specific cell population are characteristic finding in AT patients. The specific defect has been shown as an increased tendency for spontaneous breakage \& rearrangement of chromosome 2,6,7,8,14,22 \& X.24,25 .(2)Clinicalcharacteristics include progressive ataxia (100\%), telangiectasia of skin or conjunctivae $(83.8 \%)$ and of ears $(70.2 \%)$, eye movement disorder (apraxia of horizontal and vertical saccadic eye movements)(80.6\%), choreoathetosis (87.1\%), dysarthria in almost all cases.$(4,5,6)$ The index case had progressive ataxia, telangiectasia over bulbar conjunctiva and dysarthria.

\section{References:-}

1. Huang KY, Shyur SD, Wang CY, Shen EY, Liang DC. Ataxia telangiectasia: Report of two cases. J MicrobiolImmunol Infect 2001;34:71-5. Back to cited text no. 1 [PUBMED]

2. Shiloh Y. ATM (ataxia telangiectasia mutated):expanding roles in the DNA damage response andcellular homeostasis. BiochemSoc Trans. 2001; 29: 661-6.

3. Moin M, Aghamohammadi A, Kouhi A, et al. Ataxia-telangiectasia in Iran: clinical and laboratory features of 104 patients. Pediatr Neurol.2007; 37: 21-8.

4. Sardanelli F et al. Cranial MRI in ataxiatelangiectasia. Neuroradiology. 1995;37:77-82

5. Strober W, et al. Immunoglobulin metabolism inataxia telangiectasia. J Clin Invest. 1968; 47:1905.

6. Bohlega SA, Shinwari JM, Al Sharif LJ, Khalil DS, Alkhairallah TS, Al TassanNA.Clinical and molecular characterization of ataxia with oculomotor apraxia patients in Saudi Arabia. BMC Med Genet. 2011 Feb 16;12:27. 